OPEN ACCESS

Edited by:

Lisa Solieri,

Università degli Studi di Modena e

Reggio Emilia, Italy

Reviewed by:

Teresa Zotta,

University of Basilicata, Italy

Vittorio Capozzi,

Italian National Research Council, Italy

*Correspondence:

Zhenmin Liu

liuzhenmin@brightdairy.com

tThese authors have contributed equally to this work

Specialty section:

This article was submitted to

Food Microbiology,

a section of the journal

Frontiers in Microbiology

Received: 09 December 2019

Accepted: 12 May 2020

Published: 07 July 2020

Citation:

Yan M, Wang B, Fu X, Gui M, Wang G, Zhao L, Li R, You C and

Liu Z (2020) Petunidin-Based

Anthocyanin Relieves Oxygen Stress in Lactobacillus plantarum ST-III.

Front. Microbiol. 11:1211. doi: 10.3389/fmicb.2020.01211

\section{Petunidin-Based Anthocyanin Relieves Oxygen Stress in Lactobacillus plantarum ST-III}

\author{
Minghui Yan ${ }^{1 \dagger}$, Bing-hua Wang ${ }^{2+}$, Xiaofei $\mathrm{Fu}^{1}$, Min Gui ${ }^{1}$, Guojiao Wang ${ }^{3}$, Lei Zhao ${ }^{4}$, \\ Ruiying $\mathrm{Li}^{1,5}$, Chunping You ${ }^{1}$ and Zhenmin Liu ${ }^{1 *}$
}

\begin{abstract}
'State Key Laboratory of Dairy Biotechnology, Shanghai Engineering Research Center of Dairy Biotechnology, Dairy Research Institute, Bright Dairy \& Food Co., Ltd., Shanghai, China, ${ }^{2}$ Department of Clinical Laboratory, Central Laboratory, Jing'an District Center Hospital of Shanghai, Fudan University, Shanghai, China, ${ }^{3}$ Bright Farming Co., Ltd., Shanghai, China, ${ }^{4}$ School of Life Sciences, Shanghai University, Shanghai, China, ${ }^{5}$ College of Food Science and Technology, Shanghai Ocean University, Shanghai, China
\end{abstract}

Application of probiotics in the food industry has been hampered by their sensitivity to challenging conditions that reduce their vitality in food matrices. A lot of attempts have been made to promote the growth of these probiotics in the aspect of nutrition demands. Among the other adverse conditions, oxygen stress can restrict the growth of probiotics and has not yet been paid enough attention to. In this study, the effect of a petunidinbased anthocyanin (ACN) on the growth of probiotic Lactobacillus plantarum ST-III was investigated under oxygen stress. The growth of ST-III was analyzed through spot assay on agar plates as well as plating-based enumeration of the viable cells in the liquid culture. Results indicated that ACN could efficiently improve the growth of ST-III under oxygen stress, whereas no effect was observed in the absence of oxygen stress. Further investigations indicated that ACN reduced the oxido-reduction potential of the culture; meanwhile, it exerted a positive transcriptional regulation on the thioredoxin system of ST-III, leading to a decrease in reactive oxygen species accumulation within the cells. Moreover, ACN enabled the growth of ST-III in reconstituted skim milk and promoted the formation of milk clots. These results revealed the role of a petunidin-based ACN in oxygen stress relief and highlighted its potential in manufacture and preservation of $L$. plantarumbased dairy products.

\footnotetext{
Keywords: Lactobacillus plantarum, petunidin, anthocyanin, Lycium ruthenicum Murray, oxygen stress, probiotics, milk
}

\section{INTRODUCTION}

According to the expert consensus document of the International Scientific Association for Probiotics and Prebiotics (ISAPP), probiotics are currently defined as live microorganisms that, when administered in adequate amounts, confer a health benefit on the host (Hill et al., 2014). To date, randomized controlled trials have shown health benefits of probiotics across a range of populations, from healthy individuals to those with acute and chronic diseases (Huang et al., 2015; Papadimitriou et al., 2015; Mu et al., 2018). Based on these studies, probiotic products have been developed in the form of drugs, foods, or drinks such as yoghurts and 
dietary supplements. Lactobacillus and Bifidobacterium are the two main genera on the list of probiotics developed so far. Unfortunately, many of the most studied probiotic strains are oxygen-sensitive (Mills et al., 2011; Hill et al., 2014; Bhattarai et al., 2019), and the toxicity of oxygen has become a main factor that restricts the application of these oxygen-sensitive probiotics (Ruiz et al., 2012; Maresca et al., 2018). Lactobacillus plantarum is a well-documented probiotic species and several strains have shown health benefits such as cholesterol-lowering, anti-inflammatory effect, gut microbiota modulation, and protection against heavy metals (Zhai et al., 2016; Wu et al., 2017; Dan et al., 2018; Malik et al., 2018). Despite its probiotic potentials, $L$. plantarum lacks the enzymes to deal with toxic oxygen-derived compounds and therefore is sensitive to oxygen stress (Papadimitriou et al., 2016). Several studies have demonstrated that some strains are able to grow under aerobic (AE) conditions in complex and synthetic media (Pedersen et al., 2012; Guidone et al., 2013; Zotta et al., 2017); however, L. plantarum strains are generally hard to grow under milk conditions, probably due to their weak proteolytic ability (Ma et al., 2016; Behera et al., 2018). L. plantarum ST-III is a probiotic strain with a lot of beneficial effects, including cholesterol-lowering and gut microbiota modulation (Liu et al., 2008; Yan et al., 2018a). Like its counterparts, ST-III is sensitive to oxygen and fails to grow in milk under air conditions, restricting its applications in the dairy industry (Siragusa et al., 2014; Jiang et al., 2016).

Anthocyanin (ACN) is one of the subclasses of phenolic phytochemicals characterized by its antioxidant properties. Naturally occurring ACNs are mostly in the form of anthocyanidin glycosides. Chemically, anthocyanidins are grouped into 3-hydroxyanthocyanidins, 3-deoxyanthocyanidins, and $\mathrm{O}$-methylated anthocyanidins; the most common types of anthocyanidins are cyanidin, delphinidin, pelargonidin, peonidin, petunidin, and malvidin (Khoo et al., 2017). ACNs are potential pharmaceutical ingredients that give various beneficial health effects, as they possess anti-oxidative and antimicrobial activities, improve visual and neurological health, and protect against cardiovascular disease (Wallace and Giusti, 2015; Khoo et al., 2017). It is suggested that these health benefits could be attributed to the potent antioxidant properties of ACNs (Wallace and Giusti, 2015; Khoo et al., 2017). Recent studies reported the use of ACNs of different origins to improve the growth of probiotic strains, including Bifidobacterium and Lactobacillus (Sun et al., 2018; Zhu et al., 2018). However, these studies were carried out under anaerobic (AN) conditions and the effect of oxygen was not been taken into consideration. Besides, previous studies are mostly performed with sequencing-based methods, whereas culture-based analysis is required for full clarification of the interactions between ACNs and microbes. Culture-based studies on this topic will not only provide mechanistic insights into the modulation of intestinal microbiota by ACNs, but also enable solutions for growth and viability of probiotics in food stress.

This study was designed to investigate the effect of a petunidin-based ACN from Lycium ruthenicum Murray on the growth of L. plantarum ST-III under oxygen stress. As far as we are acknowledged, this is the first report on the interaction between ACNs and probiotics. Moreover, oxygen stress relief by ACN enabled the growth of ST-III in milk, indicating a potential of ACN in manufacture of L. plantarum-based dairy products.

\section{MATERIALS AND METHODS}

\section{Preparation of ACN From Lycium ruthenicum Murray}

The dried fruits of L. ruthenicum (Black goji) were obtained from a local market in Xi'ning, Qinghai, China. The procedures for preparation of $\mathrm{ACN}$ were performed according to the reported method (Yan et al., 2018c) with some modifications. In brief, $50.0 \mathrm{~g}$ of dried fruits of L. ruthenicum were extracted with $80 \%$ aqueous ethanol (vol/vol, with $0.1 \%$ formic acid) at a ratio of $1: 40(\mathrm{wt} / \mathrm{vol})$ in $50^{\circ} \mathrm{C}$ water bath for $3 \mathrm{~h}$. The extract was then filtered through a $0.45 \mu \mathrm{m}$ cellulose filter and concentrated with vacuum rotary evaporator. The concentrate was then diluted and loaded onto a column of wet-packed AB-8 resin. The column was washed with two times bed volume of deionized water and then eluted with $85 \%$ aqueous ethanol solution (vol/vol, $\mathrm{pH}$ 2.0). The effluent was collected, concentrated, and freeze-dried, affording the ACN for the following experiments.

\section{Analysis of the ACN}

The ACN was analyzed by High Performance Liquid Chromatography (HPLC) coupled to mass spectrometer and the ACN content was determined by spectrophotometric $\mathrm{pH}$ differential method as described previously (Tang et al., 2017). Briefly, $0.4 \mathrm{ml}$ of sample was individually mixed with $3.6 \mathrm{ml}$ and $0.025 \mathrm{M}$ potassium chloride $(\mathrm{pH} 1.0)$ and $0.4 \mathrm{M}$ sodium acetate solution $(\mathrm{pH} 4.5)$, and the absorbance of the mixed samples at $\mathrm{pH} 1.0$ and 4.5 were measured at $519 \mathrm{~nm}$ against distilled water as the blank by using a spectrophotometer. The ACNs content was calculated according to the previous description (Tang et al., 2017) and expressed as cyanindin-3glucoside equivalence.

\section{L. plantarum Strains}

L. plantarum ST-III (CGMCC 0847) was provided by the State Key Laboratory of Dairy Biotechnology, Bright Dairy \& Food Co., Ltd., China. The bacteria strain was routinely activated in MRS medium (Merck, Germany) at $30^{\circ} \mathrm{C}$ in a Whitley A35 Anaerobic Workstation (Don Whitley Scientific, United Kingdom) filled with a mixture gas of $\mathrm{N}_{2}: \mathrm{H}_{2}: \mathrm{CO}_{2}=85: 10: 5$ ( $\mathrm{vol} / \mathrm{vol}$ ). The activated culture was then subjected to growth analysis under oxygen stress.

\section{Spot Assays}

Spot assays were carried out on TYC plates (15.0 g/L tryptone, $5.0 \mathrm{~g} / \mathrm{L}$ yeast extract, $0.2 \mathrm{~g} / \mathrm{L} \mathrm{L}$-cystine, $0.1 \mathrm{~g} / \mathrm{L}$ sodium sulphate, $1.0 \mathrm{~g} / \mathrm{L}$ sodium chloride, $0.8 \mathrm{~g} / \mathrm{L}$ disodium phosphate anhydrous, $2.0 \mathrm{~g} / \mathrm{L}$ sodium bicarbonate, $12.0 \mathrm{~g} / \mathrm{L}$ sodium acetate anhydrous, 
$50.0 \mathrm{~g} / \mathrm{L}$ sucrose, and $15 \mathrm{~g} / \mathrm{L}$ agar, $\mathrm{pH}$ 7.4) as described previously in $\mathrm{AE}$ and $\mathrm{AN}$ conditions (Bueno et al., 2018; Yan et al., 2018b). In brief, activated culture of L. plantarum ST-III was 1:50 diluted into fresh MRS medium and cultivated anaerobically to an OD595 of $\sim 0.8$. Cells were harvested from $10 \mathrm{ml}$ culture by centrifugation at $5,000 \mathrm{rpm}$ for $2 \mathrm{~min}$. After two times wash, the cell pellets were resuspended and 10-fold serial dilutions of cell suspensions were prepared. Five microliter aliquots from each dilution were spotted onto TYC plates and incubated aerobically at $30^{\circ} \mathrm{C}$ for 3 days. Equivalent aliquots were spotted on MRS plates and incubated anaerobically at $30^{\circ} \mathrm{C}$ as control. Photos of agar plates were taken using a Molecular Imager Gel Doc XR system (Bio-Rad) equipped with a white-light trans-illuminator. The experiments were performed in triplicate (three independent biological replicates), and the representative results are shown.

\section{Liquid Culture Under Oxygen Stress}

The growth of L. plantarum under oxygen stress was performed in liquid TYC medium under AE and AN conditions, as previously described (Yan et al., 2018b). Cell pellets from overnight culture were washed three times and resuspended into $50 \mathrm{ml}$ fresh TYC medium ( $\mathrm{pH} 7.4$ ) in $250 \mathrm{ml}$ shaken flasks to an $\mathrm{OD}_{595}$ of $\sim 0.2$. The cultures were then incubated at $30^{\circ} \mathrm{C}$ under air conditions with agitation at $120 \mathrm{rpm}$ for $24 \mathrm{~h}$, and samples were taken at $0,2,4,8,12$, and $24 \mathrm{~h}$ for enumeration of viable cells in the culture. To test its effect on the growth of L. plantarum, ACN was supplemented to a final concentration of $0.025 \%$ (wt/ vol). Equivalent cultures were incubated anaerobically and the growth was compared with that under air conditions. The experiments were performed in triplicate, and the average values are shown with standard deviations.

\section{Enumeration of L. plantarum}

The number of viable cells in the culture was determined through gradient dilutions and plating-based methodology, as described previously (Ashraf and Shah, 2011), with slight modifications. In brief, samples were taken at the indicated time points and serially diluted using sterile saline $(9.0 \mathrm{~g} / \mathrm{L}$ of $\mathrm{NaCl}$ ) and plated on MRS plates. After 3 days cultivation in Whitley A35 Anaerobic Workstation at $35^{\circ} \mathrm{C}$, plates with 30-300 colonies were counted and the numbers of viable cells were calculated and expressed as $\mathrm{CFU} / \mathrm{ml}$ culture. The enumeration was carried out in triplicate and the average results are shown.

\section{ROS Determination}

L. plantarum ST-III was cultivated in TYC medium under AE or AN conditions, as described above. After $12 \mathrm{~h}$ cultivation, bacteria cells were harvested by centrifugation at 5,000 rpm for $2 \mathrm{~min}$ and the level of reactive oxygen species (ROS) was determined using 2,7-dichlorodihydrofluorescein diacetate $\left(\mathrm{H}_{2} \mathrm{DCF}-\mathrm{DA}\right.$; Beyotime Institute of Biotechnology, Haimen, China) according to the instructions provided by the manufacturer. In brief, around $1 \times 10^{6}$ cells were collected and washed with phosphate-buffered solution (0.01 M phosphate, $\mathrm{pH} 7.4, \mathrm{PBS}$ ) and then treated with $10 \mathrm{mM} \mathrm{H}_{2}$ DCF-DA dissolved in $\mathrm{PBS}$ at $37^{\circ} \mathrm{C}$ anaerobically for $20 \mathrm{~min}$. After removal of $\mathrm{H}_{2} \mathrm{DCF}-\mathrm{DA}$ and three times wash with PBS, the fluorescence intensity was monitored with excitation wavelength at $488 \mathrm{~nm}$ and emission wavelength at $525 \mathrm{~nm}$ on SpectraMax M5, Molecular Devices (San Jose, CA, United States).

For each sample, an equal amount of cells were sonicated and subjected to quantification of the total protein using the Bradford method. The fluorescence intensity was normalized with the total protein content, and the relative amount of ROS is expressed as DCF fluorescence intensity per milligram total protein, as described previously (Yan et al., 2018b). The experiments were performed in triplicate and the average values, as well as the standard deviations, are shown.

\section{Determination of Extracellular Oxido-Reduction Potential}

L. plantarum ST-III was cultivated in TYC medium under air conditions and the extracellular oxido-reduction potential (ORP) was determined as described in previous studies (Zhu et al., 2014; Huang et al., 2017). In brief, after being cultured, $20 \mathrm{ml}$ culture broth was filtered under vacuum through a $0.8-\mu \mathrm{m}$ mixed cellulose ester membrane, and the filtrate was diluted appropriately to determine the ORP. ORP of the culture was detected at 12 and $24 \mathrm{~h}$ via an oxidation-reduction electrode (Leici, Shanghai), and the values were calibrated according to the reference electrode value $\left(210 \mathrm{mV}\right.$ at $\left.37^{\circ} \mathrm{C}\right)$.

\section{RNA Extraction and Transcription Analysis (qRT-PCR)}

Bacterial cells were harvested by centrifugation at $8,000 \times g$ for $10 \mathrm{~min}$ at $4^{\circ} \mathrm{C}$ and total RNA was extracted using RNeasy Mini kits (Qiagen). cDNA synthesis was performed using the FastQuant RT kit with gDNase (Tiangen) according to the manufacturer's instructions. Quantitative real time-PCR (qRT-PCR) was performed with Taq SYBRGreen qPCR Premix on a 7500 Fast System (Applied Biosystems) according to the manufacturer's instructions. The thermo-cycling conditions were $95^{\circ} \mathrm{C}$ for $10 \mathrm{~min}$, followed by 40 cycles of $95^{\circ} \mathrm{C}$ for $15 \mathrm{~s}, 55^{\circ} \mathrm{C}$ for $30 \mathrm{~s}$, and $60^{\circ} \mathrm{C}$ for $60 \mathrm{~s}$ according to a previous study (Zotta et al., 2012). The sequences of the primers are listed in Table 1. Three independent replicates of each sample were tested and the $2^{-\Delta \Delta \mathrm{Ct}}$ method was used to calculate the expression levels of the target genes. 16S rRNA transcript was used as the internal standard for transcription analysis (Chen et al., 2019).

TABLE 1 | Primers used in quantitative real time-PCR.

\begin{tabular}{ll}
\hline Name & Nucleotide sequence \\
\hline \multirow{2}{*}{ 16S rRNA } & 5'-CGCAAGGCTGAAACTCAAAGG-3' \\
& 5'-CTGACGACAACCATGCACCAC-3' \\
trxB1 & 5'-ATGGCAAAGAGTTACGACG-3' \\
& 5'-TTCAGAACCAGTCCCAATGAC-3' \\
trxA2 & 5'-ATGGTCGCAGCAACTACTG-3' \\
& 5'-TTATAGATATTGAGCTAAAGTTG-3' \\
& 5'-ATGGAAAAACAAACAGTA-3' \\
& 5'-GCATCATCGGAGTTCGTTAAAATG-3'
\end{tabular}




\section{The Growth of L. plantarum in Reconstituted Skim Milk}

The reconstituted skim milk (RSM) was prepared, as previously described (Li et al., 2016). Skim milk powder (33.4\% protein, $0.8 \%$ fat, $54.1 \%$ lactose, and $7.9 \%$ minerals, Fonterra Ltd., Auckland, New Zealand) was reconstituted in distilled water. The RSM was stirred with a Stirrer RW20 (IKA, Staufen, Germany) at $600 \mathrm{rpm}$ for $5 \mathrm{~min}$, and then heat-treated at $95^{\circ} \mathrm{C}$ for $15 \mathrm{~min}$ immediately. After cooling to room temperature $\left(25^{\circ} \mathrm{C}\right), \mathrm{ACN}$ was supplemented to a final concentration of $0.025 \%$ (wt/vol). The mixtures were then inoculated with L. plantarum ST-III $\left(5 \times 10^{7} \mathrm{CFU} / \mathrm{ml}\right)$ and incubated aerobically in a $30^{\circ} \mathrm{C}$ incubator. Samples were taken at the indicated time points and the viable cells were quantified with a plating-based methodology as described above. During the incubation, the formation of milk clots was investigated. Photos were taken at $48 \mathrm{~h}$ and the representative images of milk clots are shown.

For spot assays on milk plates, RSM agar plates (containing $1 \%$ skimmed milk powder and $1.5 \%$ agar, wt/vol) were exploited. Cell suspensions of ST-III were 10-fold serially diluted and spotted onto RSM agar plates with or without ACN. Growth was performed under air conditions at $30^{\circ} \mathrm{C}$ and photographs of the plates were taken after $72 \mathrm{~h}$ cultivation. All the experiments were performed in triplicate (three independent biological replicates).

\section{RESULTS}

\section{A Petunidin-Based Anthocyanin From Lycium ruthenicum Murray}

The ACN was extracted from dried fruits of L. ruthenicum and purified, as described previously (Zheng et al., 2011) and the ACN was analyzed by HPLC coupled to mass spectrometer. Determination of the ACN content by $\mathrm{pH}$ differential method showed that the ACN obtained contains $3.7 \mathrm{~g} / \mathrm{L} \mathrm{ACN}$, among which nearly $98 \%$ is ACN, as revealed by HPLC analysis. This result is consistent with previous studies (Zheng et al., 2011; Yan et al., 2018c).

\section{ACN Stimulated the Growth of Lactobacillus plantarum ST-III Under Oxygen Stress}

The effect of the ACN on the growth of ST-III was investigated under oxygen stress, a condition usually encountered in the food industry. Oxygen stress was simulated as previously described (Yan et al., 2018b) and the growth of ST-III was analyzed by spot assays. As shown in Figure 1A, the growth of ST-III was much more vigorous when ACN was supplemented (compare ACN with Ctrl). The ACN could promote the growth of ST-III by about two orders of magnitude, whereas no obvious effect was observed in the AN control (Figure 1B), suggesting that the ACN could efficiently promote the growth of ST-III under oxygen stress.

\section{Effect on the Growth of L. plantarum ST-III in Liquid Culture}

The effect on the growth of $L$. plantarum ST-III was also examined in liquid TYC medium, as previously described (Yan et al., 2018b), with or without ACN. Bacterial growth was reflected by colony-forming units, and the growth of ST-III with $0.025 \% \mathrm{ACN}$ was compared to that without ACN. As shown in Figure 2, cells of ST-III in liquid culture with ACN proliferated after a 2-h stagnation, whereas those without ACN experienced a growth stagnation of about $4 \mathrm{~h}$. This trend was consistent with the oxygen stress level reported in a previous study (Yan et al., 2016). After $24 \mathrm{~h}$ cultivation, the growth advantage in ACN was still obvious. However, no such growth advantage was observed in the AN culture (Figure 2, dashed lines). These results indicated that ACN could effectively promote the growth of L. plantarum ST-III in liquid culture under oxygen stress. Note should be taken that the growth advantage endowed by ACN was not as obvious as that observed on agar plates. This might be explained by the lower intensity of oxygen stress in liquid culture, as revealed in previous studies (Raso et al., 2012; Laureys et al., 2018).

\section{Relief of the Oxygen Stress by ACN}

The results above indicated that the growth improvement might be attained through relief of the oxygen stress. The extent of
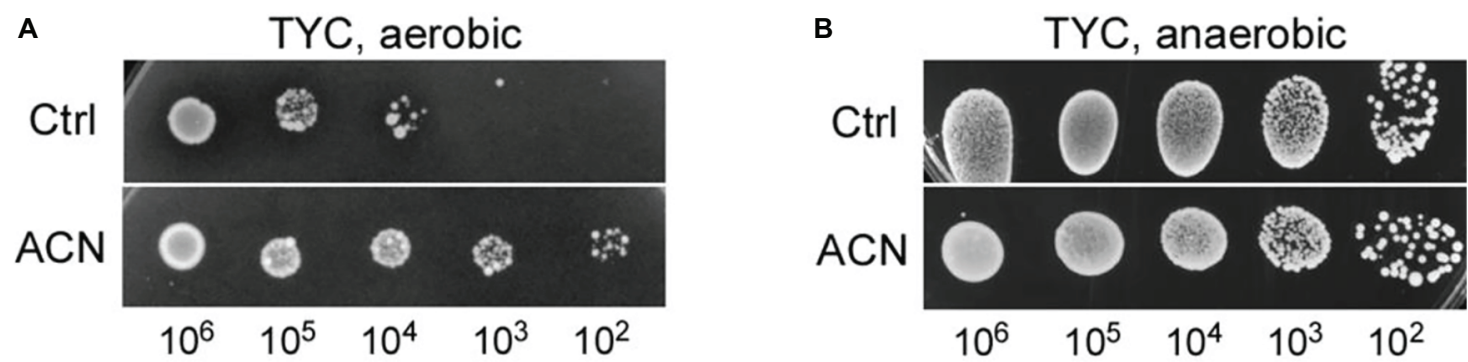

FIGURE 1 | The growth of Lactobacillus plantarum ST-III under oxygen stress as reflected by spot assays on TYC-agar plates. (A) Cells of ST-III were suspended and serially diluted, the cell suspensions were then spotted onto TYC plates with or without anthocyanin (ACN) and cultivated aerobically at $30^{\circ} \mathrm{C}$ for $48 \mathrm{~h}$. (B) For anaerobic (AN) control, equivalent plates were cultivated anaerobically. All the photographs of the agar plates were taken with a Molecular Imager Gel Doc XR system (Bio-Rad) equipped with a white-light trans-illuminator. The experiments were performed in triplicate, and the representative images are shown. 


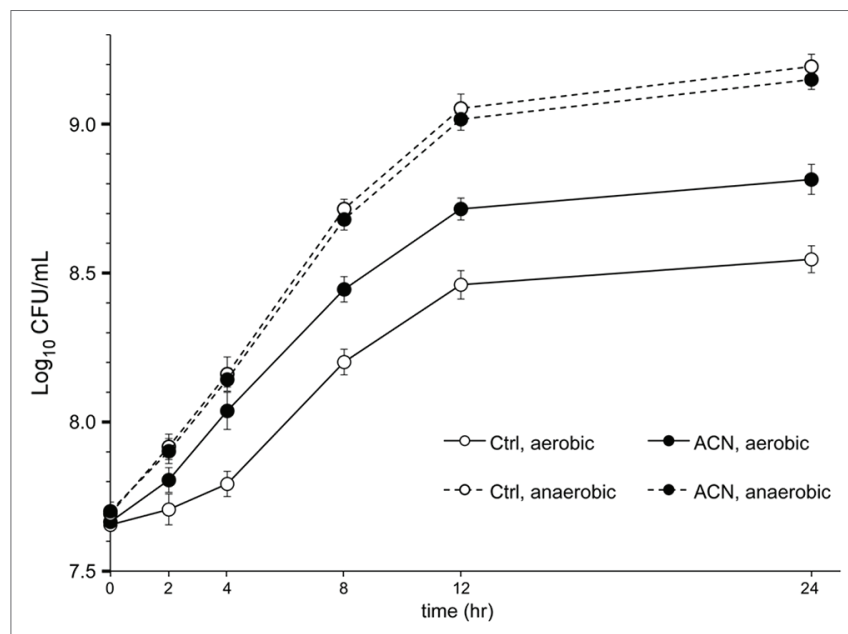

FIGURE 2 | The growth of $L$. plantarum ST-III in liquid culture. L. plantarum ST-III was cultivated in liquid TYC medium with or without $0.025 \% \mathrm{ACN}$ under aerobic (AE) and AN conditions (shown in solid and dashed lines, respectively). The growth of ST-III was estimated by the number of viable cells (CFU/ml) determined by plating-based method. Experiments were performed in triplicate and the average values, as well as the standard deviations, are included in the graphs.

oxygen stress within ST-III cells inoculated with or without ACN was analyzed. Previous studies showed that a considerable amount of ROS accumulated within cells of lactic acid bacteria encountered with oxygen stress (Guidone et al., 2013; Imlay, 2015). Therefore, ROS accumulation within cells of ST-III was quantified to evaluate the intensity of oxygen stress. As shown in Figure 3A, after $12 \mathrm{~h}$ cultivation under oxygen stress, ST-III cells accumulated ROS at $\sim 30 \mathrm{DCF} / \mathrm{mg}$ pro, whereas supplementation of $0.025 \%$ ACN could reduce the amount of ROS by more than half. This result was, in accordance with the growth advantage of ST-III with $\mathrm{ACN}$, suggesting that the growth improvement by $\mathrm{ACN}$ might have been mediated by relief of the oxygen stress.

Additionally, the ORP of the culture, an essential physiological parameter for micro-aerobic fermentations (Liu et al., 2013), was determined. The results indicated that the ORP of the medium was obviously reduced by ACN (Figure 3B), consistent with the trend of ROS accumulation (Figure 3A). These results provided further evidence that the growth improvement by ACN was attained through relief of the oxygen stress.

\section{The Involvement of Thioredoxin (TRX) System}

When encountered with oxidative stress, L. plantarum enhanced the expression of antioxidant enzymes such as those of the thioredoxin (TRX) system (Serrano et al., 2007). Previous studies identified $\operatorname{tr} x B 1$ and $\operatorname{tr} x A 2$ as two genes highly conserved within the $L$. plantarum strains that encode the main enzymes in the TRX system (Serrano et al., 2007; Zhai et al., 2020). qRT-PCR assays were performed to investigate the effect of ACN on the transcription of $\operatorname{tr} x B 1$ and $\operatorname{tr} x A 2$ in ST-III. As shown in Figure 4, both $\operatorname{tr} x A 2$ and $\operatorname{tr} x B 1$ were significantly up-regulated by ACN (by 2.87- and 4.65-fold, respectively), suggesting that the oxygen stress relief by ACN might be associated with the enhancement of TRX system. In addition, the transcription of $c c p A$, an important gene involved in the AE growth of L. plantarum (Zotta et al., 2012), was not affected.

\section{Oxygen Stress Relief by ACN Enabled the Aerobic Growth of ST-III on Milk Plates}

Although they are widely seen in a lot of fermented foods, food conditions are generally regarded as stress conditions for L. plantarum and other lactic acid bacteria (Papadimitriou et al., 2016). The effect of ACN in relief of the oxygen stress led to the hypothesis that it might improve the growth of ST-III in food stress. Therefore, the effect of ACN on the growth of ST-III was evaluated by spot assays on RSM-agar plates under air conditions. As shown in Figure 5, whereas ST-III failed to grow on RSM-agar plate (as shown in Ctrl group), the supplementation of $0.025 \%$ ACN could efficiently stimulate the growth of ST-III (shown as bacteria spots in ACN group). This result suggested that relief of the oxygen stress by ACN might bypass the nutritional demand of ST-III and enable its growth on milk plates.

\section{ACN Improved the Growth of ST-III in Liquid RSM}

Subsequently, the effect of ACN on the growth of ST-III was investigated in liquid RSM. The viable cells in the liquid RSM culture with or without ACN were enumerated. As shown in Figure 6A, when $0.025 \%$ ACN was supplemented, ST-III underwent a much more vigorous growth in liquid RSM, as compared to that without ACN. After 48 h cultivation, ST-III could proliferate by more than 10 -fold with ACN, whereas only 3-fold of proliferation was observed in the absence of ACN. However, no obvious growth improvement was observed under AN conditions (Figure 6A). Notably, after $48 \mathrm{~h}$ cultivation, milk clots formation by ST-III was evident in liquid RSM supplemented with ACN, whereas the group without ACN did not coagulate (Figure 6B). This is consistent with the proliferation of ST-III with ACN observed in Figure 6A. These results revealed that ACN is also effective in growth improvement of ST-III in liquid RSM, suggesting its potential in manufacture of L. plantarum-based dairy products.

\section{DISCUSSION}

Probiotics are defined as live microorganisms that, when administered in adequate amounts, confer a health benefit on the host (Hill et al., 2014). In order to exert a beneficial effect, a probiotic needs to be administered in sufficiently high numbers, and thus it is imperative that the probiotic survives cultivation, processing, and storage. Lactobacillus and Bifidobacterium are two important genera on the list of probiotics with well-established health benefits to human body (Hill et al., 2014; Fiocco et al., 2019). However, strains of both genera are generally sensitive to oxygen, and oxygen stress has become one of the most important abiotic factors that negatively affect their growth and survival (Papadimitriou et al., 2016; Fiocco et al., 2019). Oxygen, however, is prevalent in the process of manufacture and storage of probiotic 
A

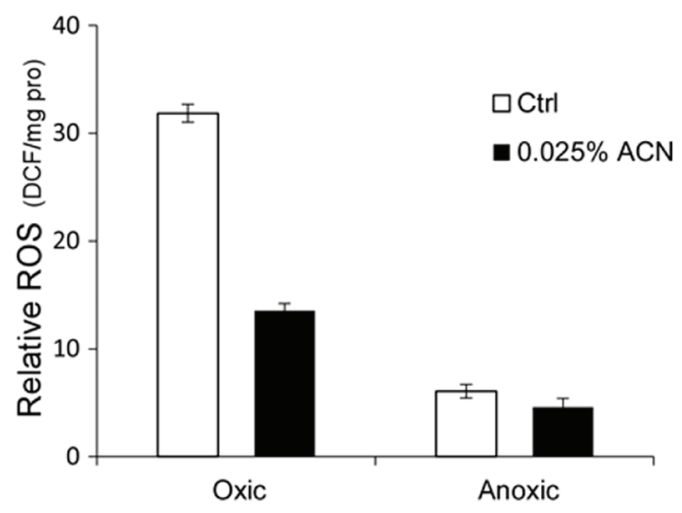

B

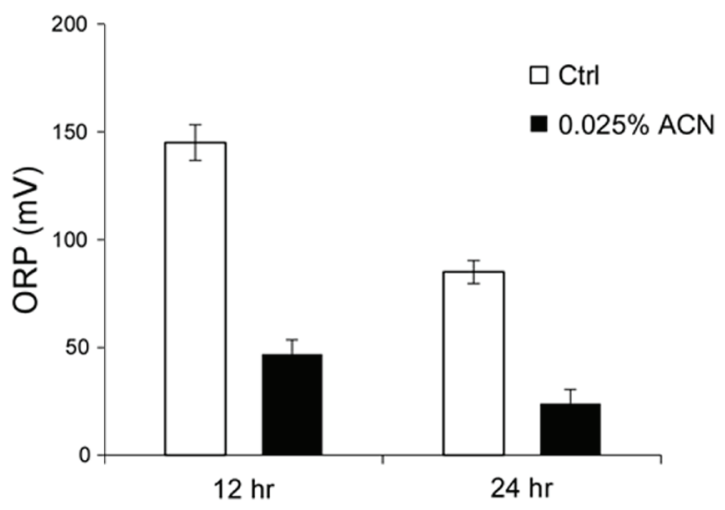

FIGURE 3 | Reactive oxygen species (ROS) accumulation within cells of ST-III and oxido-reduction potential (ORP) of the culture. (A) Determination of ROS accumulation within the cells. L. plantarum ST-III was incubated anaerobically or in the presence of environmental oxygen. Samples with or without ACN were taken after $48 \mathrm{~h}$ incubation. The amount of ROS within the cells was determined using DCFH-DA as a probe and the results were normalized to total protein content (relative ROS expressed as DCF/mg pro). (B) ORP of the medium during cultivation. Culture of ST-III was sampled at the indicated time points and ORP was determined. The experiments were performed in triplicate and the average results, as well as the standard deviations, are included in the graphs.

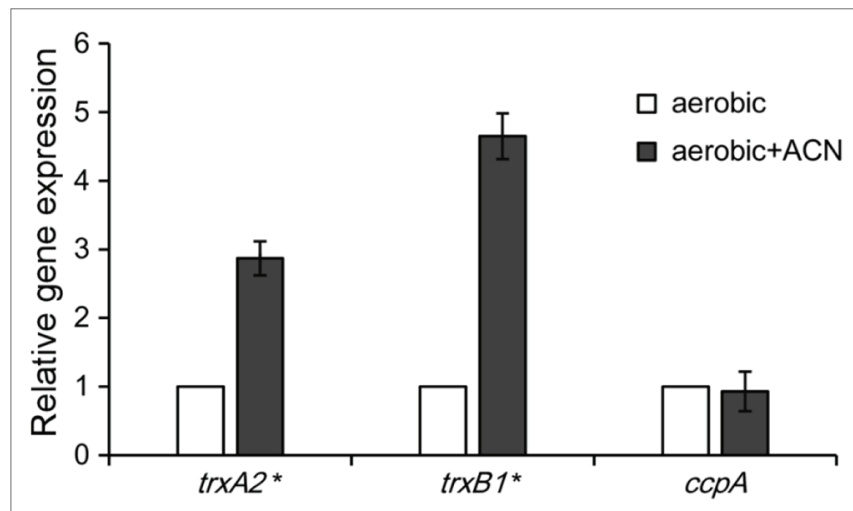

FIGURE 4 | Quantitative real time-PCR (qRT-PCR) analysis of the oxidative stress-related genes. L. plantarum ST-III was cultivated under oxygen stress with and without $A C N$ and the relative expression level of $\operatorname{tr} x A 2$, trxB1, and ccpA was measured by qRT-PCR. The transcription level of each gene with $A C N$ was expressed as fold to that without $A C N$. The results are shown as average value of three biological and technical replicates. Statistical significance is identified as ${ }^{*} p<0.05$.

products, making it an enormous threat to the performance of probiotics. Therefore, it is an urgent demand to develop techniques to protect these probiotic bacteria from oxygen toxicity.

L. plantarum is a well-documented probiotic species, and several strains have shown health benefits such as cholesterollowering, anti-inflammatory effect, gut microbiota modulation, and protection against heavy metals (Zhai et al., 2016; Wu et al., 2017; Dan et al., 2018). L. plantarum is a lactic acid bacterium found in diverse ecological niches, highlighting its particular capabilities of adaptation (Seddik et al., 2017). Therefore, it is employed as a model organism for academic research of lactic acid bacteria (Siezen and van Hylckama Vlieg, 2011; Seddik et al., 2017; Parlindungan et al., 2019), also in the area of stress response and tolerance (Fiocco et al., 2010; Papadimitriou

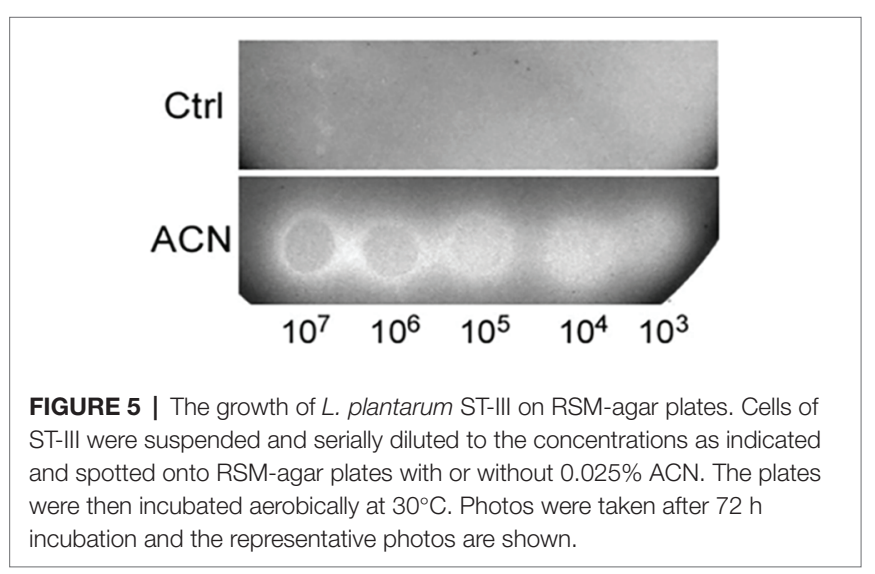

et al., 2016; Arena et al., 2019; Zhao et al., 2019). Studies on L. plantarum have deepened the understanding of oxygentolerance in Lactobacillus and other probiotics (Archibald and Fridovich, 1981; Serrano et al., 2007; Papadimitriou et al., 2016).

The growth of probiotic L. plantarum in milk has become a critical issue in the dairy industry that hampers their extensive applications. According to previous studies, the growth of these probiotics in milk is restricted by factors including nutrients inadequacy, acid, and oxygen stress (Li et al., 2016; Fiocco et al., 2019). Moreover, this problem is further complicated by the interactions among these growth-restricting factors (Papadimitriou et al., 2016; Zhang et al., 2018).

ACN is one of the subclasses of phenolic phytochemicals widely spread in flowers, fruits, and vegetables with a wide variety of biological activities (Wallace and Giusti, 2015). Biochemical studies indicated that these health benefits are associated with the antioxidant properties of ACN compounds (Zheng et al., 2011; Wallace and Giusti, 2015). Plant-derived ACNs have been used as functional ingredients in dietary and health food supplements. The involvement of ACNs in the 

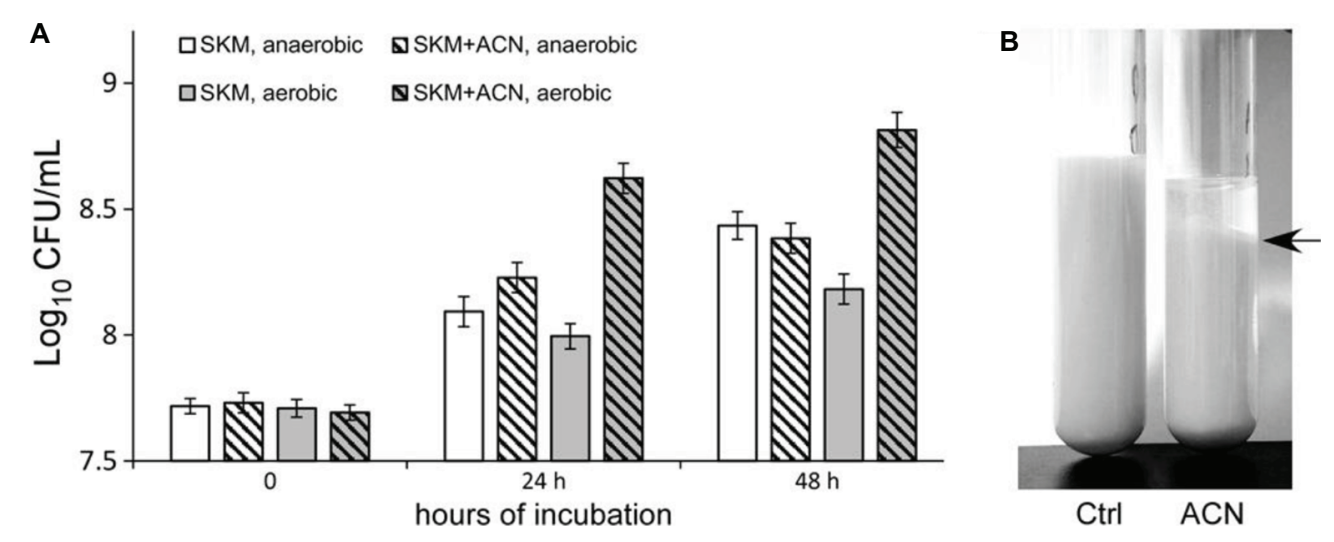

FIGURE 6 | The growth of L. plantarum ST-III in liquid reconstituted skim milk (RSM). (A) L. plantarum ST-III was cultivated in liquid RSM under AE and AN conditions. The growth of ST-III was estimated by CFU/ml. Experiments were performed in triplicate, and the average values, as well as the standard deviations were included in the graphs. A slight inhibition of growth was observed; this might be attributed to the weak antimicrobial activity of ACN, as previous studies have shown the anti-microbial activity of polyphenol compounds (Daglia, 2012; Bouarab-Chibane et al., 2019). (B) Representative image of ST-III culture in RSM after 48 h AE incubation. Note that milk clot formation in ST-III culture with ACN was obvious (indicated by a black arrow on the right).

modulation of intestinal microbiota has been analyzed by $16 \mathrm{~S}$ rDNA sequence-based analyses (Chen et al., 2018; Yan et al., 2018c). The results indicated that ACNs significantly increased the relative abundances of Bifidibacterium and Allisonella (Yan et al., 2018c). A few recent studies indicated that ACNs from berries could reverse the dextran sulfate sodium-induced imbalance in gut microbiota (Chen et al., 2018; Peng et al., 2019). However, the mechanisms that underlie the impact of ACNs on the microbiota remain to be clarified, and in vitro culture-based analysis is required for full clarification of the interactions between ACNs and microbes.

This study performed an in vitro, culture-based analysis of the microbiological effects of ACN from L. ruthenicum Murray, a unique nutritional food widely distributed in the salinized desert of Qinghai-Tibet Plateau. As far as we are acknowledged, it is the first in vitro study on the interaction between probiotics and ACNs. Oxygen stress relief observed in this study might provide an explanation for ACN-induced increase of Lactobacillus, Bifidobacterium (Chen et al., 2018; Peng et al., 2019), and other anaerobes such as Akkermansia (Cremonini et al., 2019) in intestinal microbiota. Meanwhile, the TRX system is a key component in the antioxidant system widely distributed in lactic acid bacteria, and the enhancement of the TRX system by ACN implies its potential in growth improvement of other oxygen-sensitive probiotics.

Moreover, from the perspective of the food industry, this study provides a solution to the growth of probiotic L. plantarum in milk. The effect of ACN can be considered for industrial

\section{REFERENCES}

Archibald, F. S., and Fridovich, I. (1981). Manganese and defenses against oxygen toxicity in Lactobacillus plantarum. J. Bacteriol. 145, 442-451. doi: 10.1128/JB.145.1.442-451.1981

Arena, M. P., Capozzi, V., Longo, A., Russo, P., Weidmann, S., Rieu, A., et al. (2019). The phenotypic analysis of Lactobacillus plantarum shsp mutants purposes and has practical implications for commercial applications of L. plantarum and other probiotics in the dairy industry.

\section{DATA AVAILABILITY STATEMENT}

The raw data supporting the conclusions of this article will be made available by the authors, without undue reservation, to any qualified researcher.

\section{AUTHOR CONTRIBUTIONS}

MY, BW, and ZL designed the experiments, analyzed the results, and wrote the manuscript. MY, BW, and XF performed the experiments with the assistance of $\mathrm{LZ}$ and RL. MG, GW and CY provided technique assistance and valuable suggestions for this project. All authors reviewed and approved the final version of the manuscript.

\section{FUNDING}

This study was supported by Shanghai Engineering Center of Dairy Biotechnology (No. 19DZ2281400), the Rising-Star Project from Science and Technology Commission of Shanghai Municipality (No. 19QB1400300), and the Joint Medical Research Program of Shanghai Jing'an District Science and Technology Commission and Health Commission (No. 2017QN01). 
Behera, S. S., Ray, R. C., and Zdolec, N. (2018). Lactobacillus plantarum with functional properties: an approach to increase safety and shelf-life of fermented foods. Biomed. Res. Int. 2018:9361614. doi: 10.1155/2018/9361614

Bhattarai, S., Cassarini, C., and Lens, P. N. L. (2019). Physiology and distribution of archaeal methanotrophs that couple anaerobic oxidation of methane with sulfate reduction. Microbiol. Mol. Biol. Rev. 83, e00074-e00018. doi: 10.1128/ mmbr.00074-18

Bouarab-Chibane, L., Forquet, V., Lanteri, P., Clement, Y., Leonard-Akkari, L., Oulahal, N., et al. (2019). Antibacterial oroperties of polyphenols: characterization and QSAR (quantitative structure-activity relationship) models. Front. Microbiol. 10:829. doi: 10.3389/fmicb.2019.00829

Bueno, E., Sit, B., Waldor, M. K., and Cava, F. (2018). Anaerobic nitrate reduction divergently governs population expansion of the enteropathogen Vibrio cholerae. Nat. Microbiol. 3, 1346-1353. doi: 10.1038/s41564-018-0253-0

Chen, L., Jiang, B., Zhong, C., Guo, J., Zhang, L., Mu, T., et al. (2018). Chemoprevention of colorectal cancer by black raspberry anthocyanins involved the modulation of gut microbiota and SFRP2 demethylation. Carcinogenesis 39, 471-481. doi: 10.1093/carcin/bgy009

Chen, C., Wang, L., Lu, Y., Yu, H., and Tian, H. (2019). Comparative transcriptional analysis of Lactobacillus plantarum and its ccpA-knockout mutant under galactooligosaccharides and glucose conditions. Front. Microbiol. 10:1584. doi: $10.3389 /$ fmicb.2019.01584

Cremonini, E., Daveri, E., Mastaloudis, A., Adamo, A. M., Mills, D., Kalanetra, K., et al. (2019). Anthocyanins protect the gastrointestinal tract from high fat diet-induced alterations in redox signaling, barrier integrity and dysbiosis. Redox Biol. 26:101269. doi: 10.1016/j.redox.2019.101269

Daglia, M. (2012). Polyphenols as antimicrobial agents. Curr. Opin. Biotechnol. 23, 174-181. doi: 10.1016/j.copbio.2011.08.007

Dan, T., Chen, H., Li, T., Tian, J., Ren, W., Zhang, H., et al. (2018). Influence of Lactobacillus plantarum P-8 on fermented milk flavor and storage stability. Front. Microbiol. 9:3133. doi: 10.3389/fmicb.2018.03133

Fiocco, D., Capozzi, V., Collins, M., Gallone, A., Hols, P., Guzzo, J., et al. (2010). Characterization of the CtsR stress response regulon in Lactobacillus plantarum. J. Bacteriol. 192, 896-900. doi: 10.1128/JB.01122-09

Fiocco, D., Longo, A., Arena, M. P., Russo, P., Spano, G., and Capozzi, V. (2019). How probiotics face food stress: they get by with a little help. Crit. Rev. Food Sci. Nutr. 60, 1552-1580. doi: 10.1080/10408398.2019.1580673

Guidone, A., Ianniello, R. G., Ricciardi, A., Zotta, T., and Parente, E. (2013). Aerobic metabolism and oxidative stress tolerance in the Lactobacillus plantarum group. World J. Microbiol. Biotechnol. 29, 1713-1722. doi: 10.1007/ s11274-013-1334-0

Hill, C., Guarner, F., Reid, G., Gibson, G. R., Merenstein, D. J., Pot, B., et al. (2014). Expert consensus document. The International Scientific Association for probiotics and prebiotics consensus statement on the scope and appropriate use of the term probiotic. Nat. Rev. Gastroenterol. Hepatol. 11, 506-514. doi: $10.1038 /$ nrgastro.2014.66

Huang, T., Tan, H., Lu, F., Chen, G., and Wu, Z. (2017). Changing oxidoreduction potential to improve water-soluble yellow pigment production with Monascus ruber CGMCC 10910. Microb. Cell Factories 16:208. doi: 10.1186/ s12934-017-0828-0

Huang, R., Tao, X., Wan, C., Li, S., Xu, H., Xu, F., et al. (2015). In vitro probiotic characteristics of Lactobacillus plantarum ZDY 2013 and its modulatory effect on gut microbiota of mice. J. Dairy Sci. 98, 5850-5861. doi: $10.3168 /$ jds.2014-9153

Imlay, J. A. (2015). Diagnosing oxidative stress in bacteria: not as easy as you might think. Curr. Opin. Microbiol. 24, 124-131. doi: 10.1016/j.mib.2015.01.004

Jiang, M., Zhang, F., Wan, C., Xiong, Y., Shah, N. P., Wei, H., et al. (2016). Evaluation of probiotic properties of Lactobacillus plantarum WLPL04 isolated from human breast milk. J. Dairy Sci. 99, 1736-1746. doi: 10.3168/jds.2015-10434

Khoo, H. E., Azlan, A., Tang, S. T., and Lim, S. M. (2017). Anthocyanidins and anthocyanins: colored pigments as food, pharmaceutical ingredients, and the potential health benefits. Food Nutr. Res. 61:1361779. doi: $10.1080 / 16546628.2017 .1361779$

Laureys, D., Aerts, M., Vandamme, P., and De Vuyst, L. (2018). Oxygen and diverse nutrients influence the water kefir fermentation process. Food Microbiol. 73, 351-361. doi: 10.1016/j.fm.2018.02.007

Li, S., Gong, G., Ma, C., Liu, Z., and Cai, J. (2016). Study on the influence of tea extract on probiotics in skim milk: from probiotics propagation to metabolite. J. Food Sci. 81, M1981-M1986. doi: 10.1111/1750-3841.13383
Liu, Z. D., Guo, B. H., Wang, Y. Y., and Wu, Z. J. (2008). Cholesterol removal from media by Lactobacillus plantarum ST-III. Milchwissenschaft 63, 134-137.

Liu, C. G., Xue, C., Lin, Y. H., and Bai, F. W. (2013). Redox potential control and applications in microaerobic and anaerobic fermentations. Biotechnol. Adv. 31, 257-265. doi: 10.1016/j.biotechadv.2012.11.005

Ma, C., Cheng, G., Liu, Z., Gong, G., and Chen, Z. (2016). Determination of the essential nutrients required for milk fermentation by Lactobacillus plantarum. LWT Food Sci. Technol. 65, 884-889. doi: 10.1016/j.lwt.2015.09.003

Malik, M., Suboc, T. M., Tyagi, S., Salzman, N., Wang, J., Ying, R., et al. (2018). Lactobacillus plantarum $299 \mathrm{v}$ supplementation improves vascular endothelial function and reduces inflammatory biomarkers in men with stable coronary artery disease. Circ. Res. 123, 1091-1102. doi: 10.1161/CIRCRESAHA.118.313565

Maresca, D., Zotta, T., and Mauriello, G. (2018). Adaptation to aerobic environment of Lactobacillus johnsonii/gasseri strains. Front. Microbiol. 9:157. doi: 10.3389/ fmicb.2018.00157

Mills, S., Stanton, C., Fitzgerald, G. F., and Ross, R. (2011). Enhancing the stress responses of probiotics for a lifestyle from gut to product and back again. Microb. Cell Factories 10:S19. doi: 10.1186/1475-2859-10-S1-S19

Mu, Q., Tavella, V. J., and Luo, X. M. (2018). Role of Lactobacillus reuteri in human health and diseases. Front. Microbiol. 9:757. doi: 10.3389/fmicb.2018.00757

Papadimitriou, K., Alegria, A., Bron, P. A., De Angelis, M., Gobbetti, M., Kleerebezem, M., et al. (2016). Stress physiology of lactic acid bacteria. Microbiol. Mol. Biol. Rev. 80, 837-890. doi: 10.1128/MMBR.00076-15

Papadimitriou, K., Zoumpopoulou, G., Foligne, B., Alexandraki, V., Kazou, M., Pot, B., et al. (2015). Discovering probiotic microorganisms: in vitro, in vivo, genetic and omics approaches. Front. Microbiol. 6:58. doi: 10.3389/fmicb.2015.00058

Parlindungan, E., May, B. K., and Jones, O. A. H. (2019). Metabolic insights into the effects of nutrient stress on Lactobacillus plantarum B21. Front. Mol. Biosci. 6:75. doi: 10.3389/fmolb.2019.00075

Pedersen, M. B., Gaudu, P., Lechardeur, D., Petit, M. A., and Gruss, A. (2012). Aerobic respiration metabolism in lactic acid bacteria and uses in biotechnology. Annu. Rev. Food Sci. Technol. 3, 37-58. doi: 10.1146/annurev-food-022811-101255

Peng, Y., Yan, Y., Wan, P., Chen, D., Ding, Y., Ran, L., et al. (2019). Gut microbiota modulation and anti-inflammatory properties of anthocyanins from the fruits of Lycium ruthenicum Murray in dextran sodium sulfate-induced colitis in mice. Free Radic. Biol. Med. 136, 96-108. doi: 10.1016/j.freeradbiomed.2019.04.005

Raso, S., Van Genugten, B., Vermuë, M., and Wijffels, R. H. (2012). Effect of oxygen concentration on the growth of Nannochloropsis sp. at low light intensity. J. Appl. Phycol. 24, 863-871. doi: 10.1007/s10811-011-9706-z

Ruiz, L., Gueimonde, M., Ruas-Madiedo, P., Ribbera, A., De Los ReyesGavilan, C. G., Ventura, M., et al. (2012). Molecular clues to understand the aerotolerance phenotype of Bifidobacterium animalis subsp. lactis. Appl. Environ. Microbiol. 78, 644-650. doi: 10.1128/AEM.05455-11

Seddik, H. A., Bendali, F., Gancel, F., Fliss, I., Spano, G., and Drider, D. (2017). Lactobacillus plantarum and its probiotic and food potentialities. Probiotics Antimicrob Proteins 9, 111-122. doi: 10.1007/s12602-017-9264-Z

Serrano, L. M., Molenaar, D., Wels, M., Teusink, B., Bron, P. A., De Vos, W. M., et al. (2007). Thioredoxin reductase is a key factor in the oxidative stress response of Lactobacillus plantarum WCFS1. Microb. Cell Factories 6:29. doi: 10.1186/1475-2859-6-29

Siezen, R. J., and Van Hylckama Vlieg, J. E. (2011). Genomic diversity and versatility of Lactobacillus plantarum, a natural metabolic engineer. Microb. Cell Factories 10(Suppl 1), S3. doi: 10.1186/1475-2859-10-S1-S3

Siragusa, S., De Angelis, M., Calasso, M., Campanella, D., Minervini, F., Di Cagno, R., et al. (2014). Fermentation and proteome profiles of Lactobacillus plantarum strains during growth under food-like conditions. J. Proteomics 96, 366-380. doi: 10.1016/j.jprot.2013.11.003

Sun, H., Zhang, P., Zhu, Y., Lou, Q., and He, S. (2018). Antioxidant and prebiotic activity of five peonidin-based anthocyanins extracted from purple sweet potato (Ipomoea batatas (L.) Lam.). Sci. Rep. 8:5018. doi: 10.1038/s41598-018-23397-0

Tang, J., Yan, Y., Ran, L., Mi, J., Sun, Y., Lu, L., et al. (2017). Isolation, antioxidant property and protective effect on PC12 cell of the main anthocyanin in fruit of Lycium ruthenicum Murray. J. Funct. Foods 30, 97-107. doi: 10.1016/j. jff.2017.01.015

Wallace, T. C., and Giusti, M. M. (2015). Anthocyanins. Adv. Nutr. 6, 620-622. doi: $10.3945 /$ an.115.009233

Wu, G., Xiao, X., Feng, P., Xie, F., Yu, Z., Yuan, W., et al. (2017). Gut remediation: a potential approach to reducing chromium accumulation using Lactobacillus plantarum TW1-1. Sci. Rep. 7:15000. doi: 10.1038/s41598-017-15216-9 
Yan, M., Chen, W., Li, N., Ren, J., Chen, C., You, C., et al. (2018a). Impact of Lactobacillus plantarum ST-III on the composition of infant gut microbiota and its potential synergism with breast milk and infant formula as revealed by an in vitro study. Int. Dairy J. 85, 66-72. doi: 10.1016/j.idairyj.2018.05.014

Yan, M., Han, J., Xu, X., Liu, L., Gao, C., Zheng, H., et al. (2016). Gsy, a novel glucansucrase from Leuconostoc mesenteroides, mediates the formation of cell aggregates in response to oxidative stress. Sci. Rep. 6:38122. doi: 10.1038/srep38122

Yan, Y., Peng, Y., Tang, J., Mi, J., Lu, L., Li, X., et al. (2018c). Effects of anthocyanins from the fruit of Lycium ruthenicum Murray on intestinal microbiota. J. Funct. Foods 48, 533-541. doi: 10.1016/j.jff.2018.07.053

Yan, M., Wang, B.-H., Xu, X., Der Meister, T., Tabyač, H.-T., Hwang, F.-F., et al. (2018b). Extrusion of dissolved oxygen by exopolysaccharide from Leuconostoc mesenteroides and its implications in relief of the oxygen stress. Front. Microbiol. 9:2467. doi: 10.3389/fmicb.2018.02467

Zhai, Q., Tian, F., Zhao, J., Zhang, H., Narbad, A., and Chen, W. (2016). Oral administration of probiotics inhibits absorption of the heavy metal Cadmium by protecting the intestinal barrier. Appl. Environ. Microbiol. 82:4429. doi: 10.1128/AEM.00695-16

Zhai, Z., Yang, Y., Wang, H., Wang, G., Ren, F., Li, Z., et al. (2020). Global transcriptomic analysis of Lactobacillus plantarum CAUH2 in response to hydrogen peroxide stress. Food Microbiol. 87:103389. doi: 10.1016/j. fm.2019.103389

Zhang, C., Lu, J., Yang, D., Chen, X., Huang, Y., and Gu, R. (2018). Stress influenced the aerotolerance of Lactobacillus rhamnosus hsryfm 1301. Biotechnol. Lett. 40, 729-735. doi: 10.1007/s10529-018-2523-6

Zhao, H., Liu, L., Peng, S., Yuan, L., Li, H., and Wang, H. (2019). Heterologous expression of argininosuccinate synthase from Oenococcus oeni enhances the acid resistance of Lactobacillus plantarum. Front. Microbiol. 10:1393. doi: $10.3389 /$ fmicb. 2019.01393

Zheng, J., Ding, C., Wang, L., Li, G., Shi, J., Li, H., et al. (2011). Anthocyanins composition and antioxidant activity of wild Lycium ruthenicum Murr. from
Qinghai-Tibet Plateau. Food Chem. 126, 859-865. doi: 10.1016/j. foodchem.2010.11.052

Zhu, Y., Li, D., Bao, G., Wang, S., Mao, S., Song, J., et al. (2014). Metabolic changes in Klebsiella oxytoca in response to low oxidoreduction potential, as revealed by comparative proteomic profiling integrated with flux balance analysis. Appl. Environ. Microbiol. 80, 2833-2841. doi: 10.1128/AEM.03327-13

Zhu, Y., Sun, H., He, S., Lou, Q., Yu, M., Tang, M., et al. (2018). Metabolism and prebiotics activity of anthocyanins from black rice (Oryza sativa L.) in vitro. PLoS One 13:e0195754. doi: 10.1371/journal.pone.0195754

Zotta, T., Parente, E., and Ricciardi, A. (2017). Aerobic metabolism in the genus Lactobacillus: impact on stress response and potential applications in the food industry. J. Appl. Microbiol. 122, 857-869. doi: 10.1111/jam.13399

Zotta, T., Ricciardi, A., Guidone, A., Sacco, M., Muscariello, L., Mazzeo, M. F., et al. (2012). Inactivation of $c c p A$ and aeration affect growth, metabolite production and stress tolerance in Lactobacillus plantarum WCFS1. Int. J. Food Microbiol. 155, 51-59. doi: 10.1016/j.ijfoodmicro.2012.01.017

Conflict of Interest: MY, XF, MG, CY, and ZL are employed by Bright Dairy \& Food Co., Ltd. GW was employed by Bright Farming Co., Ltd.

The remaining authors declare that the research was conducted in the absence of any commercial or financial relationships that could be construed as a potential conflict of interest.

Copyright (c) 2020 Yan, Wang, Fu, Gui, Wang, Zhao, Li, You and Liu. This is an open-access article distributed under the terms of the Creative Commons Attribution License (CC BY). The use, distribution or reproduction in other forums is permitted, provided the original author(s) and the copyright owner(s) are credited and that the original publication in this journal is cited, in accordance with accepted academic practice. No use, distribution or reproduction is permitted which does not comply with these terms. 